\title{
A simplified method for implementing run-time polymorphism in Fortran95
}

\author{
Viktor K. Decyk ${ }^{\mathrm{a}, \mathrm{b}}$ and Charles D. Norton ${ }^{\mathrm{a}}$ \\ ${ }^{a}$ Jet Propulsion Laboratory, California Institute of Technology 4800 Oak Grove Drive Pasadena, CA 91109-8099, \\ USA \\ ${ }^{\mathrm{b}}$ Department of Physics and Astronomy University of California, Los Angeles CA, 90095-1547, USA
}

\begin{abstract}
This paper discusses a simplified technique for software emulation of inheritance and run-time polymorphism in Fortran95. This technique involves retaining the same type throughout an inheritance hierarchy, so that only functions which are modified in a derived class need to be implemented.
\end{abstract}

\section{Introduction}

Fortran 95 is modern language primarily used for high performance in scientific and technical computing. It is an object-based language, in our opinion, which directly supports many of the important concepts in object-oriented programming, including the creation of a hierarchy of objects, information hiding and encapsulation, and static polymorphism. It is not considered an object-oriented language, however, because it does not directly support the concepts of inheritance and run-time polymorphism. These concepts can be emulated, however, and in earlier papers [1,2], we showed how to do so. This allowed one to implement an object-oriented programming style in Fortran 95. The emulation of run-time polymorphism involved the creation of a special polymorphic class. This was straightforward to implement if the inheritance hierarchy was simple. However, it was cumbersome if the inheritance hierarchy was complex. In this paper, we show a simplified method for emulating run-time polymorphism. All of the methods discussed here also work equally well in the older standard, Fortran 90.

\section{Classes}

Why are such object-oriented concepts important for scientific programming? A class is defined to be a data type along with the functions that operate on that type. The main value of classes is to encapsulate and hide the complex details of a set of related operations while presenting a simplified set of functions for the programmer to use. This encapsulation prevents inadvertent modification of internal data and also allows the implementation details to be changed without impacting the usage of the class. As a result, it is much easier for different programmers to implement different classes without getting in each others way, and it (usually) means that once a class is debugged, one does not have to worry about it further when debugging other new code. As a result, more complex computational projects can be attempted.

In our earlier paper, we used a stopwatch class as an example, first introduced by Gray and Roberts [3]. The class was intended to provide a simple means for timing parts of a program. It's usage is very simple. After first constructing a stopwatch, as follows: 
type (stopwatch) : : sw

call new_stopwatch(sw)

One can then turn the stopwatch on and off in various parts of the program, such as:

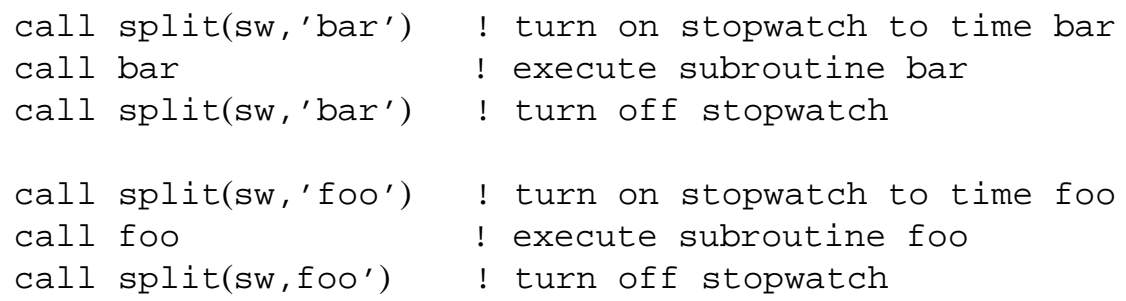

One can then report the results of these timings:

call report $(\mathrm{sw}, 6)$

where 6 refers to the output device. When the timings are done, delete the stopwatch:

call delete(sw)

Thus the class provides four functions (sometimes called methods) for the user, a constructor, a destructor, a split function and a report function. The details of what is inside the stopwatch type and how these functions are implemented do not need to be known by the user.

Of course, the person responsible for implementing this and related classes, needs to know these details. For example, one could define the class data members to be:

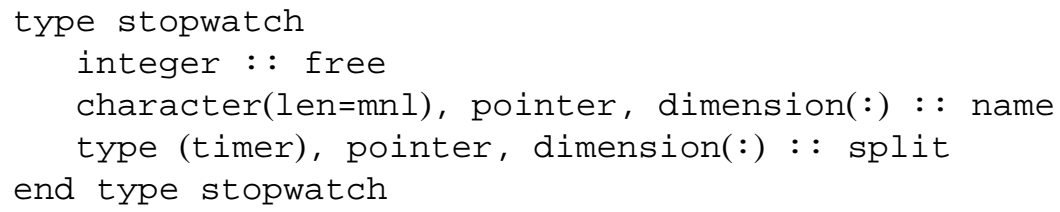

The integer data member free is used to keep track of how many stopwatches are active at any time. The data member name is an array of character strings that contains the name of each stopwatch, typically, the subroutine being timed. The data member split is an array of derived type elements that contains the current and elapsed time for each stopwatch.

\section{Inheritance}

Inheritance is used to create a hierarchy of related classes that have the same functions but different behavior. The child classes typically add data elements to the data of the parent's class and modify some but not all of the functions of the parent. Since the child class (also known as the derived class) always contains the data of the parent class (also known as the base class), it can execute the unmodified functions as if it were a parent. The type of the child class in such a case is also called a subtype of the base class. The main value of inheritance is to be able to create a set of related classes without duplicating existing code, while maintaining the same interfaces, that is, the same functions and arguments.

In an earlier paper [2], we created a parallel_stopwatch class which "inherited" from stopwatch. The parallel stopwatch was intended to provide additional functionality for timing parallel programs. In particular, it provided the global maximum time measured across the processors rather than the individual time on each processor. Since Fortran 95 does not support inheritance as a language mechanism, we emulated the functionality of inheritance by first defining a new child type that contained within it, an object of the parent type. We added a new data item, idproc, to this type to keep track of the processor id for each parallel process. The new type was defined as follows: 


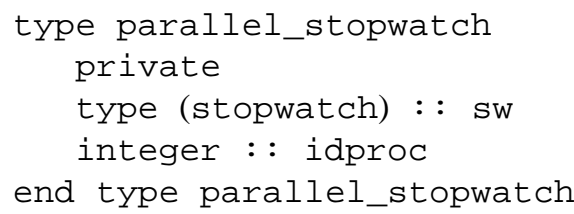

! base class component

! processor id

The inheritance relation requires that the child type provides the same functions as the parent, but the child could modify the functions, if desired. In this example, two of the four functions of stopwatch needed to be modified, the constructor and the report function. The new constructor is implemented by calling the constructor of the base class to construct the base class component sw, and a new procedure gidproc to obtain the processor id, as follows:

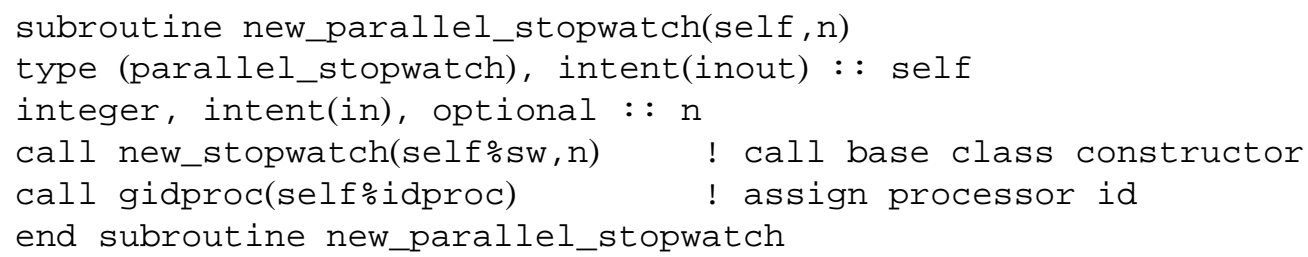

The modified report function for the stopwatch class is implemented by first calling the normal stopwatch, then calling a parallel maximum function before reporting the results. For functions in the child class which are modified, the process here is very similar to the process used in object-oriented languages. In object-oriented languages, however, unmodified functions defined in the base class automatically work in the derived class. This is not true in Fortran95, which does not allow one to use one type in place of another.

In this example, the other two functions in the parallel_stopwatch class, the destructor and the split method, did not need to be modified. In Fortran95, one needs to write a procedure with exactly one executable line which merely calls the base class procedure on the base class component of the derived type. For the split function, for example, one writes:

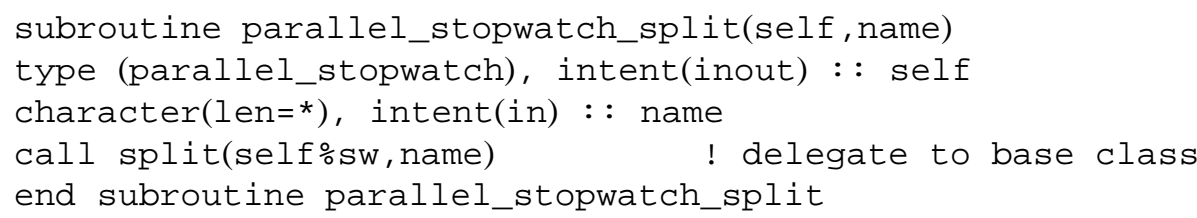

In addition, one can overload the names of the functions so that they work with both types. For example, one overloads the name split as follows:

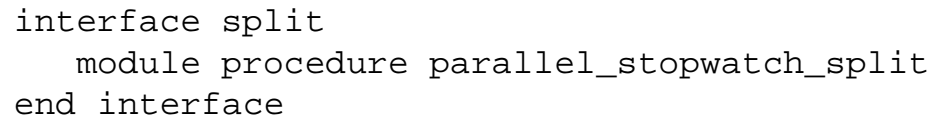

After overloading the names, the usage of the parallel stopwatch is exactly the same as the usage of the stopwatch shown earlier. Note that the child class did not have to reimplement any of the functions of the parent class. Except for the case where the child class inherits many unmodified functions, emulation of inheritance in Fortran95 is quite straightforward.

\section{Run-time polymorphism}

Run-time polymorphism (also known as dynamic binding) allows a single object name to refer to any member of an inheritance hierarchy and permits a procedure to resolve at run-time which actual object is being referred to. This is useful because it allows one to write programs in terms of a single type which would behave differently depending on the actual type. Object-oriented languages support this behavior. Fortran95 does not, with the exception of elemental functions. 
In order to emulate run-time polymorphism in Fortran95, two features must be constructed. The first feature is an identity mechanism to keep track of the actual class of an object in an inheritance hierarchy. The second is a dispatch mechanism (or method lookup) which selects the appropriate procedure to execute based on the actual class of the object.

In our earlier papers [1,2], we implemented the identity mechanism by defining a special polymorphic type consisting of pointers to all the possible types in the inheritance hierarchy, as follows:

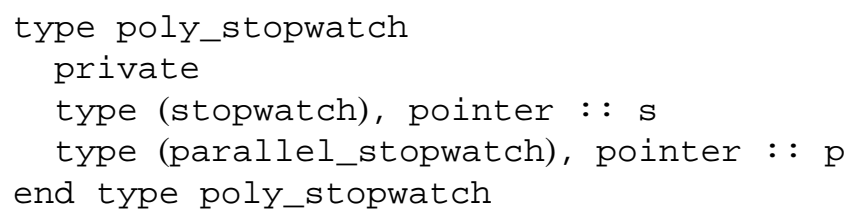

At any given time, one of the pointers is associated with an actual object, the other pointers will be set to null objects. This polymorphic type functions like a base class pointer in $\mathrm{C}++$. An assignment function was created to set the polymorphic type to one of the possible actual types. For example, to set the polymorphic type to a stopwatch type, one creates the function:

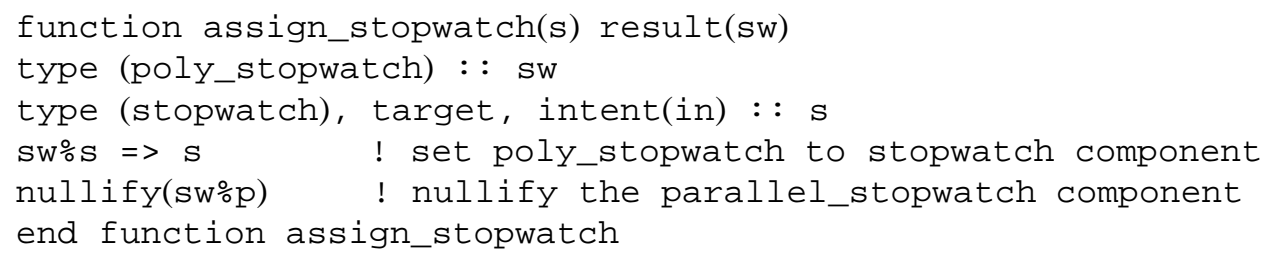

To simplify usage, we overload the assign functions with the name poly, as follows:

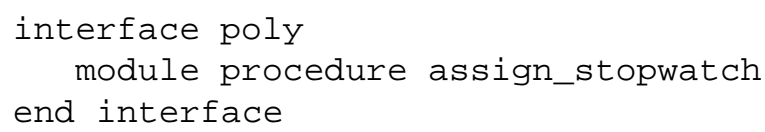

To implement the dispatch mechanism, one checks which of the possible pointers actually points to an object, then passes the associated pointer to the appropriate procedure. For example, the polymorphic split procedure would look like the following:

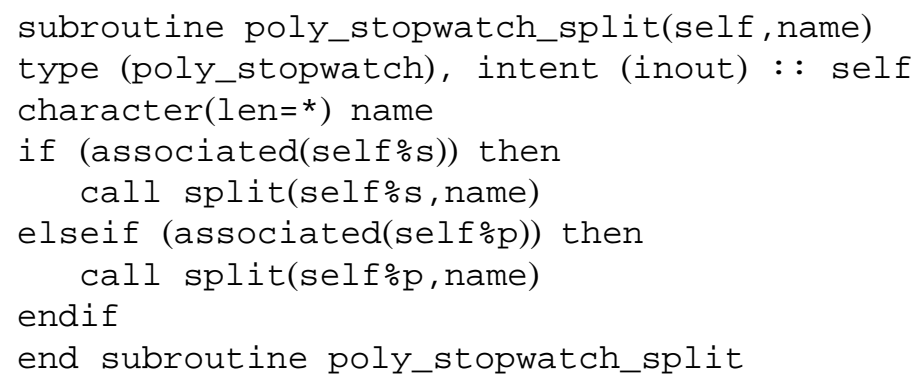

The following example illustrates how to use run-time polymorphism:

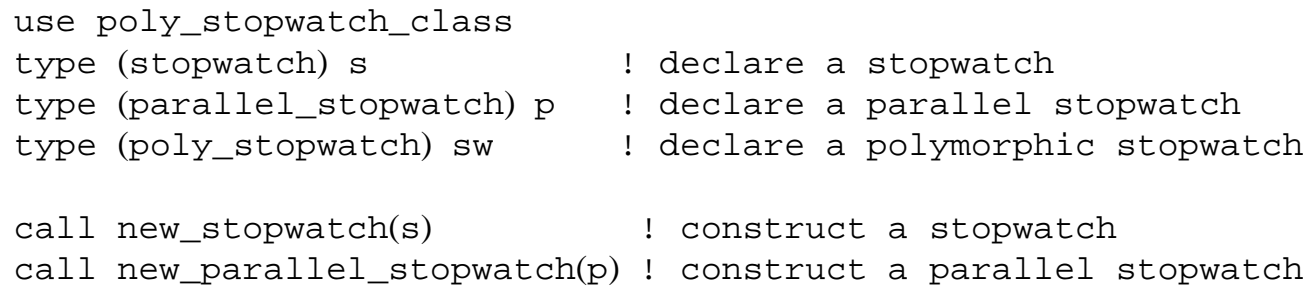




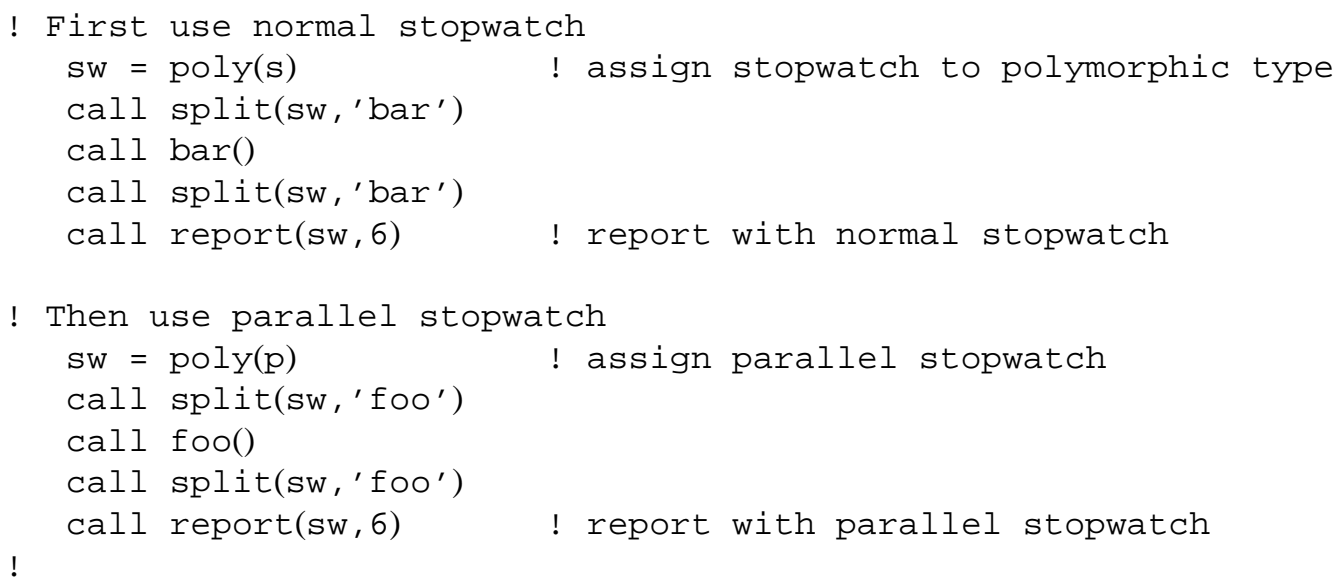

Implementing the polymorphic class is straightforward. One does not need to know the implementation details of these functions, just their argument types. In principle, the class can be implemented by a software tool. However, implementing it can be tedious if the hierarchy is deep. In particular, one must implement a dispatch mechanism for every function, even functions such as split, which are not modified by any class. Once created, however, the polymorphic class is used just like an object-oriented language such as $\mathrm{C}++$ uses the base class pointer, and the user of the hierarchy does not need to know the implementation details.

\section{Simplified emulation of run-time polymorphism}

The reason emulating run-time polymorphism can be tedious is that Fortran95 is so strict about types, and our emulation was being faithful to the subtyping mechanisms used in object-oriented languages such as $\mathrm{C}++$ or Java. Subtyping is easy in these languages, but not easy in Fortran95. If we abandon the unnatural use of subtyping in Fortran95, however, we discover that run-time polymorphism can be implemented much more simply, at the cost of giving up some encapsulation.

Instead of creating a different type for each member of the inheritance hierarchy, and a special polymorphic type to implement dynamic dispatch, we will create a single expanded type for all of them, and use a different identity and dispatch mechanism to implement run-time polymorphism. This type includes the sum total of all the data members of every class in the inheritance hierarchy, as well as an extra flag, subtype, which identifies the particular member of the inheritance hierarchy using this type. Thus for the stopwatch class hierarchy, we define the expanded type as follows:

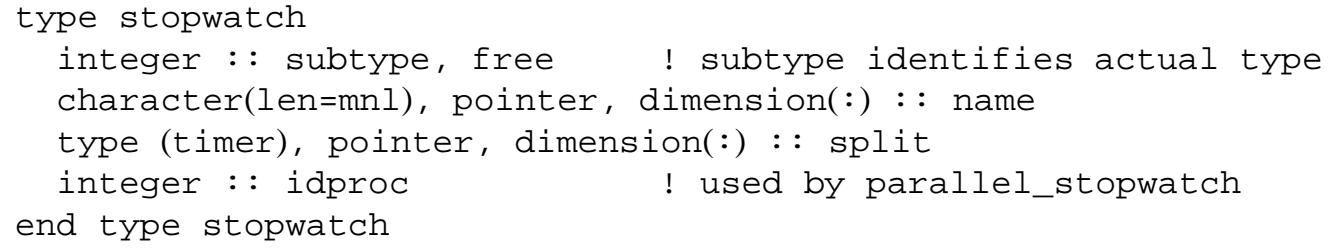

Compared to the earlier definition of stopwatch, we have two extra members, subtype to identify the actual type, and idproc, which was used by the parallel_stopwatch class. This type must know about the data members of the entire hierarchy and must be modified whenever a new class in the inheritance hierarchy is created. It can function like a base-class pointer in $\mathrm{C}++$. This type can be created in its own module, if desired.

The constructor for the stopwatch class requires an extra line, to set the value of subtype:

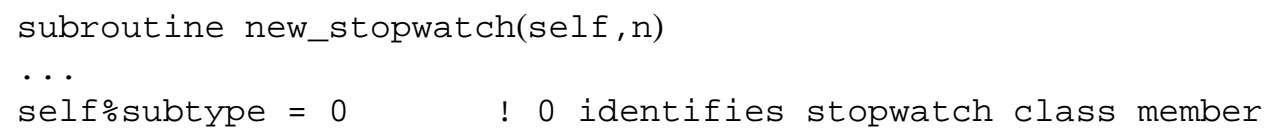


No further changes need to be made to the stopwatch class.

Implementing the inheritance hierarchy is simplified. The parallel stopwatch class no longer has a separate type. Its constructor only needs to set the subtype component (to a different value):

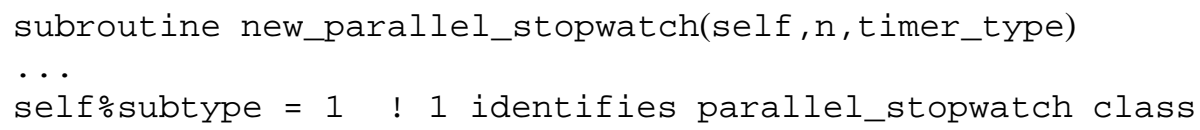

The unmodified functions, destructor and the split, no longer have to be implemented as subroutines with one executable line. This is now more like inheritance in $\mathrm{C}++$. The modified report function remains the same. However, the specific name parallel_stopwatch report is used instead of the overloaded name "report," since one no longer has different types to disambiguate the names. However, one can use the renaming facility in Fortran95 to control the names more exactly, if desired.

To implement polymorphism, we still create a polymorphic class module, but we no longer need a polymorphic type. A dispatch mechanism needs to be created only for the functions which were modified in the inheritance hierarchy. In this case, only the report function needs to have a dispatch mechanism. The subtype data member is used to select which actual function to call, as follows:

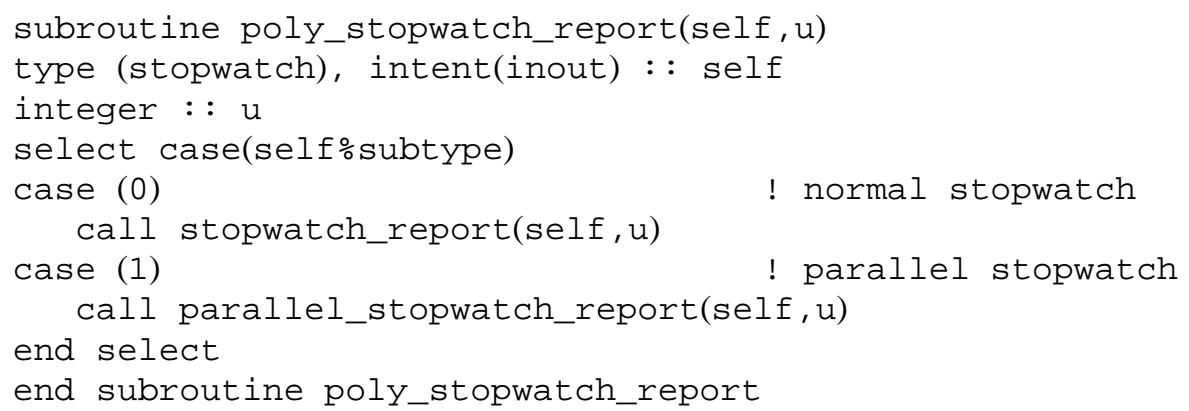

The other functions are either unmodified, or have unique names (the constructor). Since no separate type is used, no functions to assign the polymorphic types are necessary. Appendix I shows the entire inheritance hierarchy. Note that only functions which are actually modified or need to be polymorphic have to be implemented.

The following example illustrates how the simplified run-time polymorphism is used:

use poly_stopwatch_class

type (stopwatch), target : : s, p ! declare stopwatches

type (stopwatch), pointer : : sw ! declare a base class pointer

call new_stopwatch(s) ! construct a stopwatch

call new_parallel_stopwatch(p) ! construct a parallel stopwatch 
The fact that the type needs to know about the data members in the entire inheritance hierarchy is a violation of the usual encapsulation of data members in object-oriented languages. However, most Fortran programmers will find this a small price to pay for the simplicity of the implementation.

An approach similar to this involving geometric shapes was discussed by Stroustrup [4]. He calls this approach a "mess" because "adding a new shape involves 'touching' the code of every important operation on shapes." This is not the case here, since we separate out the polymorphism from the individual implementations. The polymorphic functions only need to know about the interfaces of the individual subtypes, nothing about their implementation. Adding a new subtype involves only changing the contents of the type itself, perhaps a line or two, and adding two lines of code to each polymorphic function. Changing the type, however, will force a recompilation of the entire class. Fortran programs are rarely so long, however, that this is a serious problem.

\section{Application to plasma simulation}

We have used this method of emulating run-time polymorphism in a Fortran95 based Framework designed to support parallel Particle-in-Cell (PIC) codes in plasma physics [5]. Such codes integrate self-consistently the trajectories of millions (now even billions) of particles in the electromagnetic fields created by the particles themselves. The basic structure of PIC codes[6] is to calculate a charge and/or current density from the particle co-ordinates, solve Maxwell's equations (or a subset) for the electric and/or magnetic fields, then advance the particles using Newton's law and the Lorentz force. The purpose of the Frameworks is to provide easy to use, trusted, components that are useful for building different kinds of PIC codes.

There are a variety of PIC codes in use. The most basic includes only the Coulomb electric field (solving only Poisson's equation). Other PIC codes include magnetic fields and solve either the full set of Maxwell's equation or the Darwin subset. We used our simplified method of modeling inheritance to implement a polymorphic particle type, as follows:

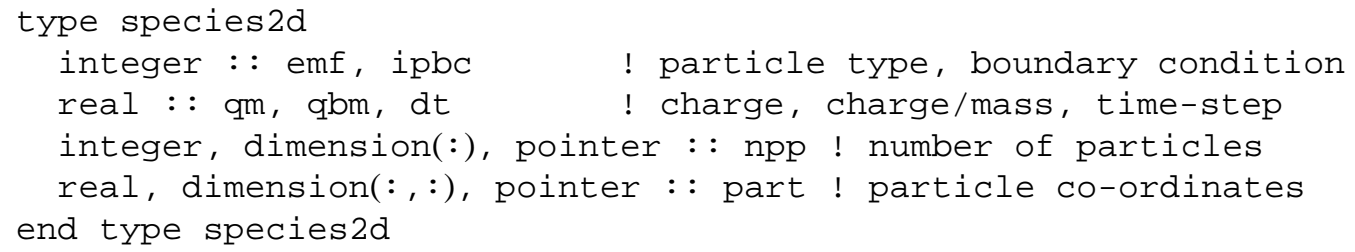

The data member emf is used to distinguish different classes of particles, namely electrostatic, magnetized electrostatic, and electromagnetic. The base class is the electrostatic type, which includes only a charge deposit and a push subroutine which uses only electric forces. The magnetized electrostatic class modifies the push subroutine to include magnetic forces. The electromagnetic class adds a current deposit method to the hierarchy. Two polymorphic methods were added which used the emf data member to determine which push subroutine was used and whether the current deposit was actually called.

\section{Discussion and conclusion}

This new method of emulation of polymorphism is attractive because of its simplicity, but it has two shortcomings. First, it results in a type which includes information not needed by some classes. In particular, it can result in an object of a base class containing some large unneeded data item from a derived class which is not used. To avoid this problem, we recommend that large data elements not be placed in such types, but rather pointers to such elements, which can be allocated when needed.

Another problem is that the data elements in a class are no longer private, and thus can be accessed and modified directly by any other class. In our own work, we never access the data elements directly outside of the class hierarchy where they are defined, so in fact, encapsulation is preserved. However, the language does not enforce this, as it does in object-oriented languages, so it is up to the programmer to preserve it. It is possible in principle to make the 
elements of the data type private and provide accessor functions for each data element but we doubt most Fortran programmers, who tend to be performance conscious, would tolerate the overhead. Finally, if enforcing such privacy is important, one can always revert back to our older method [1,2]. The older method is also useful when trying to add classes to a hierarchy whose internal details are not well understood or whose source code is unavailable. Programmers can use either method, depending on the situation.

\section{Appendix I: Inheritance hierarchy for stopwatch classes}

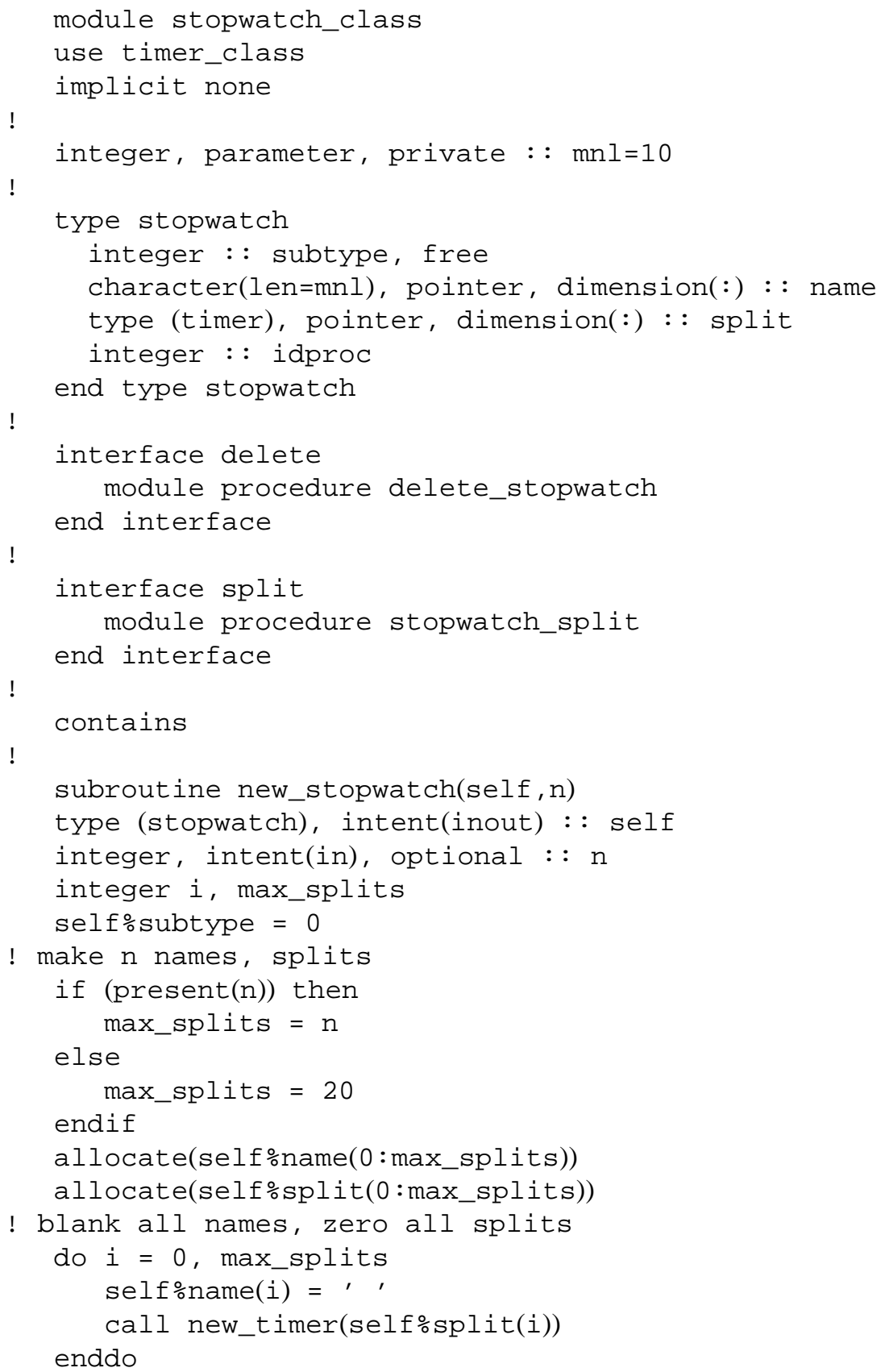




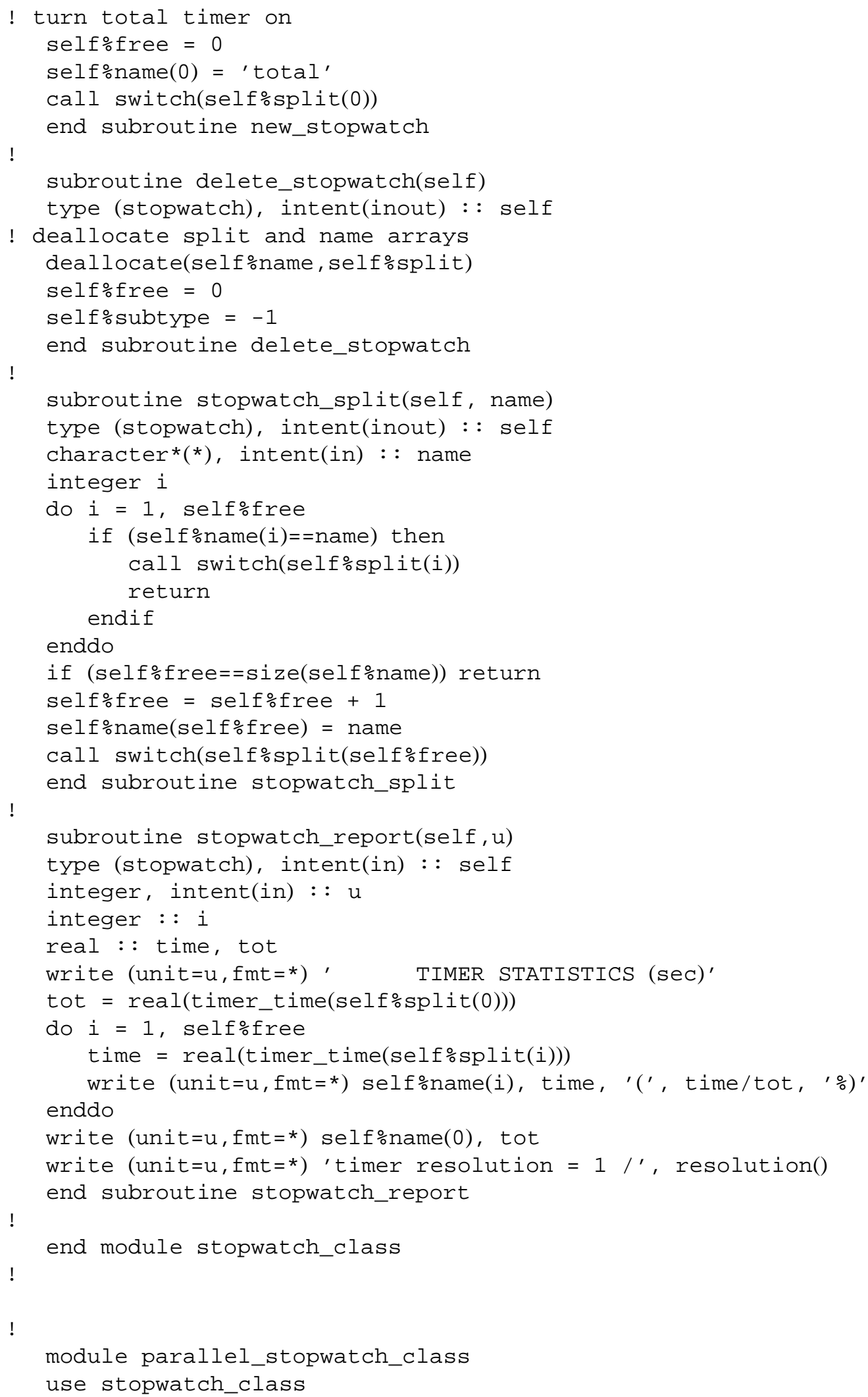




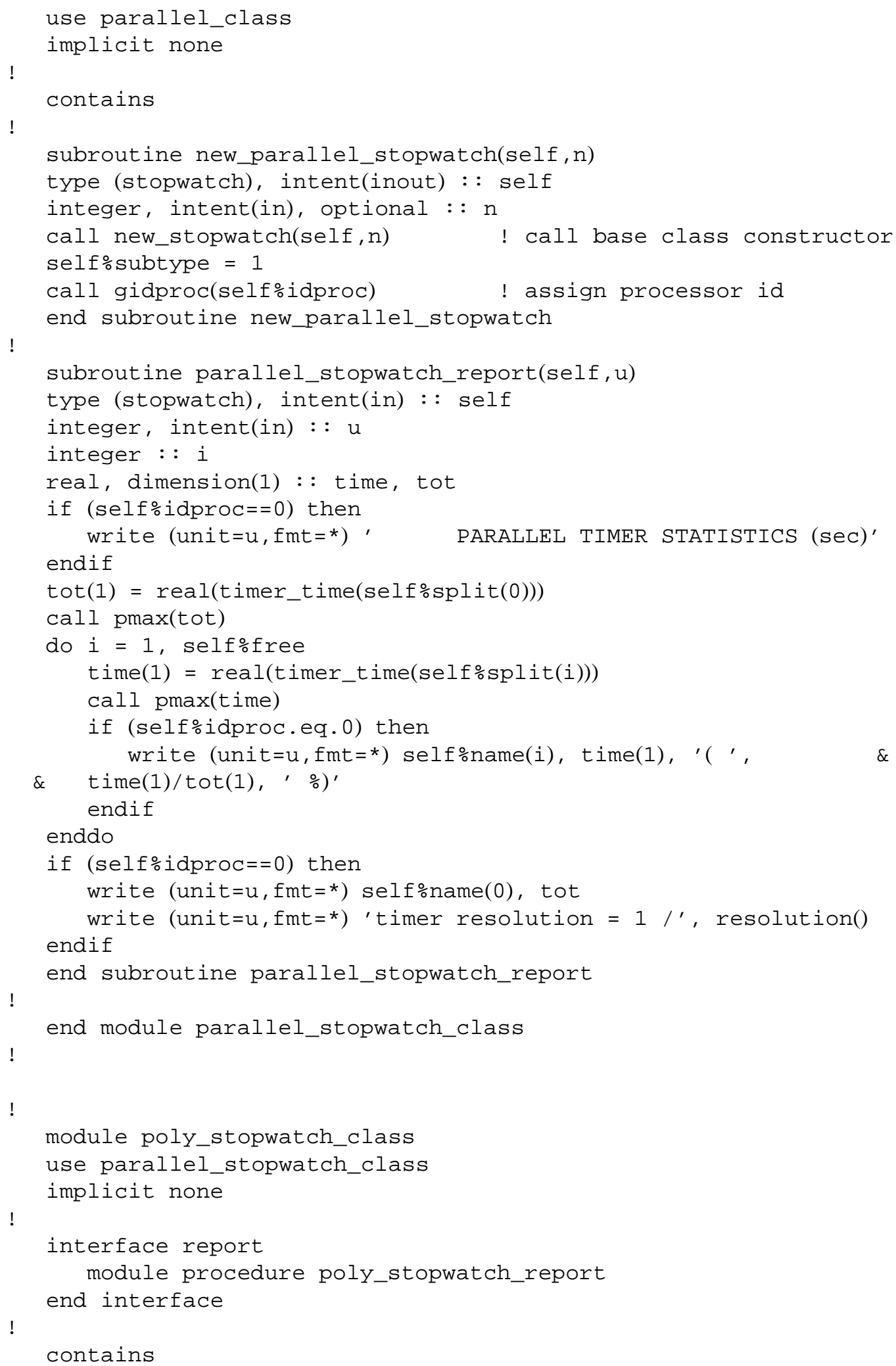




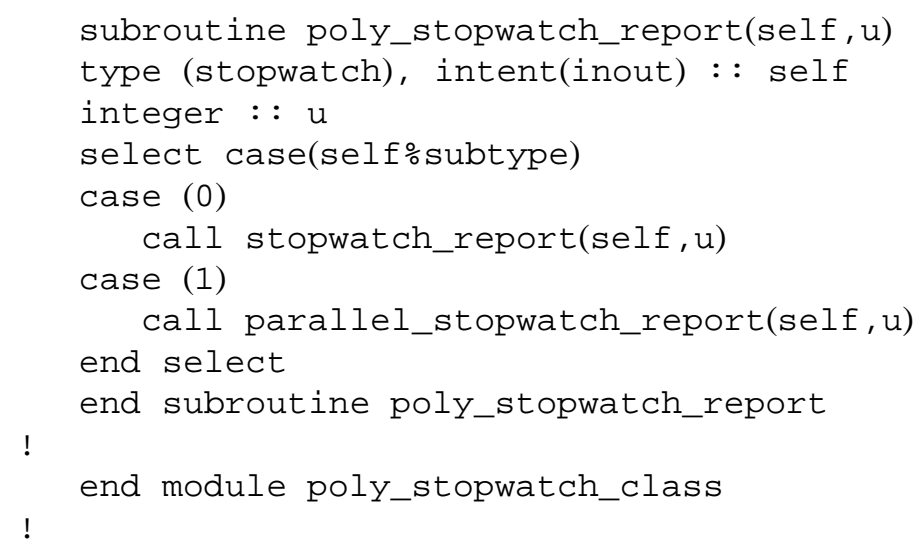

\section{Acknowledgments}

This research was carried out in part at the Jet Propulsion Laboratory, California Institute of Technology, under a contract with the national Aeronautics and Space Administration. The research of V. K. D. was also supported by the US Department of Energy, under the SCIDAC program.

\section{References}

[1] V.K. Decyk, C.D. Norton and B.K. Szymanski, How to Express C++ Concepts in Fortran 90, Scientific Programming 6(4) (1997), 363.

[2] V.K. Decyk, C.D. Norton and B.K. Szymanski, How to Support Inheritance and Run-Time Polymorphism in Fortran 90, Computer Physics Communications 115(9) (1998).

[3] M.G. Gray and R.M. Roberts, Object-Based Programming in Fortran 90, Computers in Physics 11(355) (1997).

[4] Bjarne Stroustrup, The C++ Programming Language, Addison-Wesley, Reading, MA, 1997, pp. 37-38.

[5] C. Huang, V. Decyk, S. Wang, E.S. Dodd, C. Ren, W.B. Mori, A Parallel Particle-in-Cell Code for Efficiently Modeling Plasma Wakefield Accleration: QuickPIC, Proc. of the 18th Annual Review of Progress in Applied Computational Electromagnetics, Monterey, CA, March, 2002, pp. 557.

[6] C.K. Birdsall and A.B. Langdon, Plasma Physics via Computer Simulation, Adam Hilger, New York, 1991. 

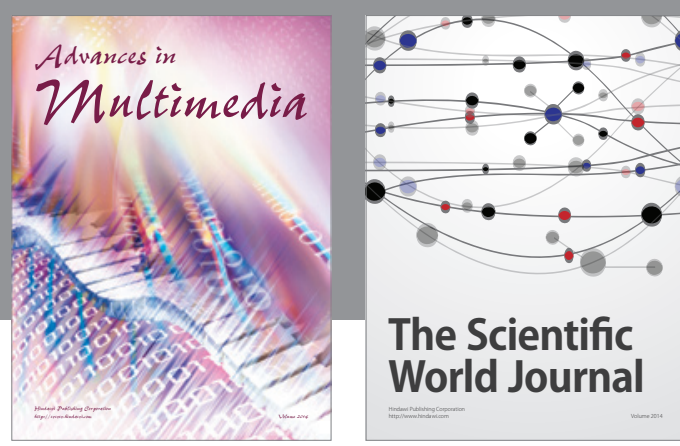

The Scientific World Journal
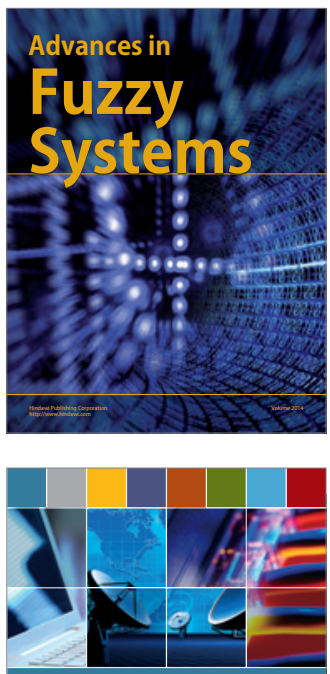

Computer Networks and Communications
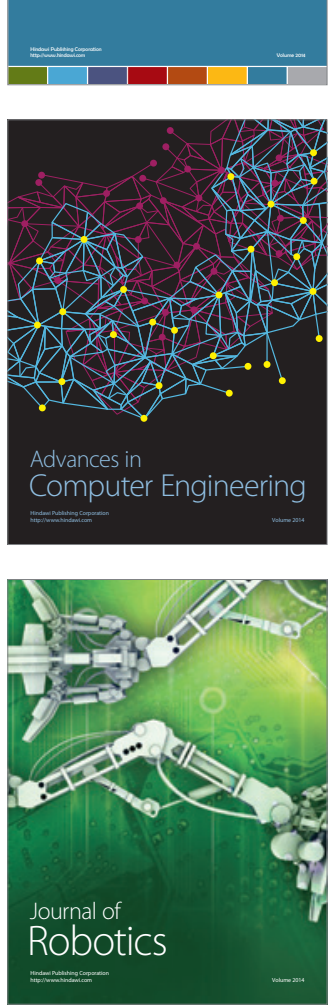
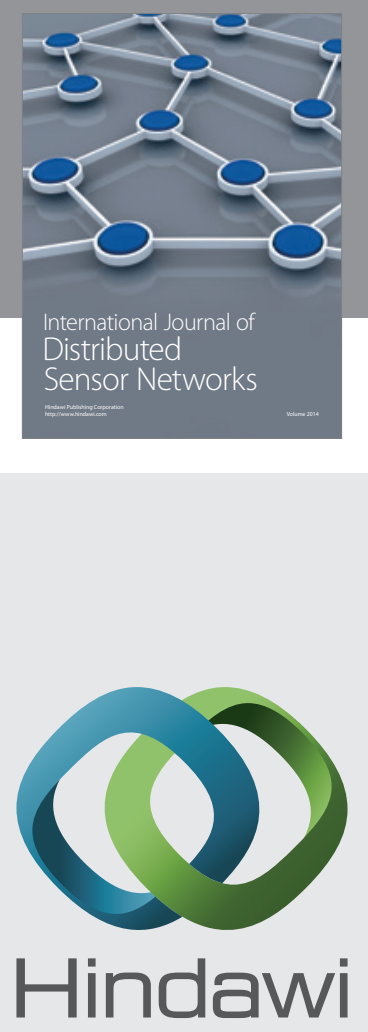

Submit your manuscripts at

http://www.hindawi.com
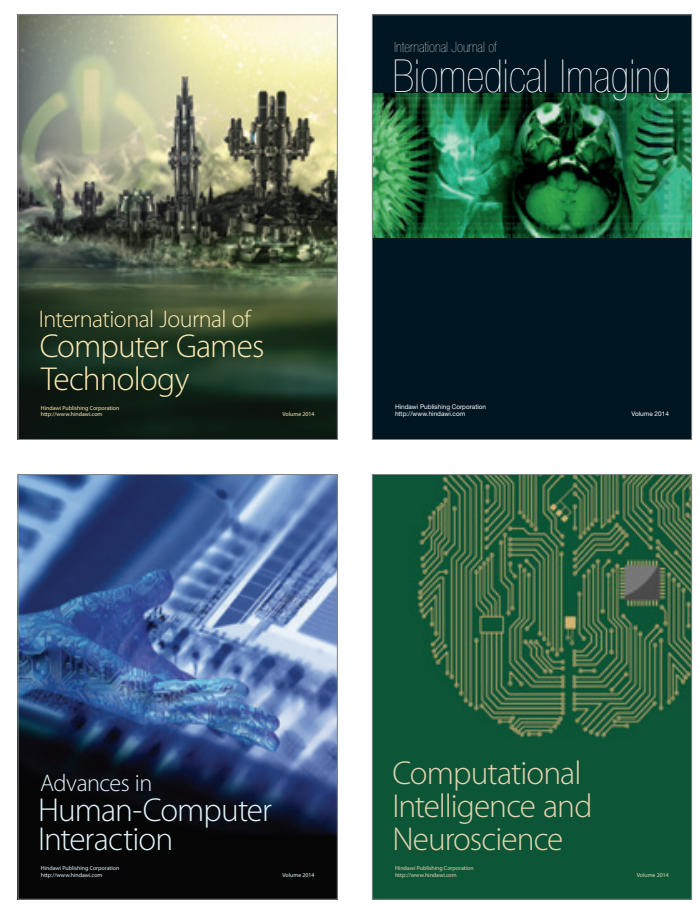
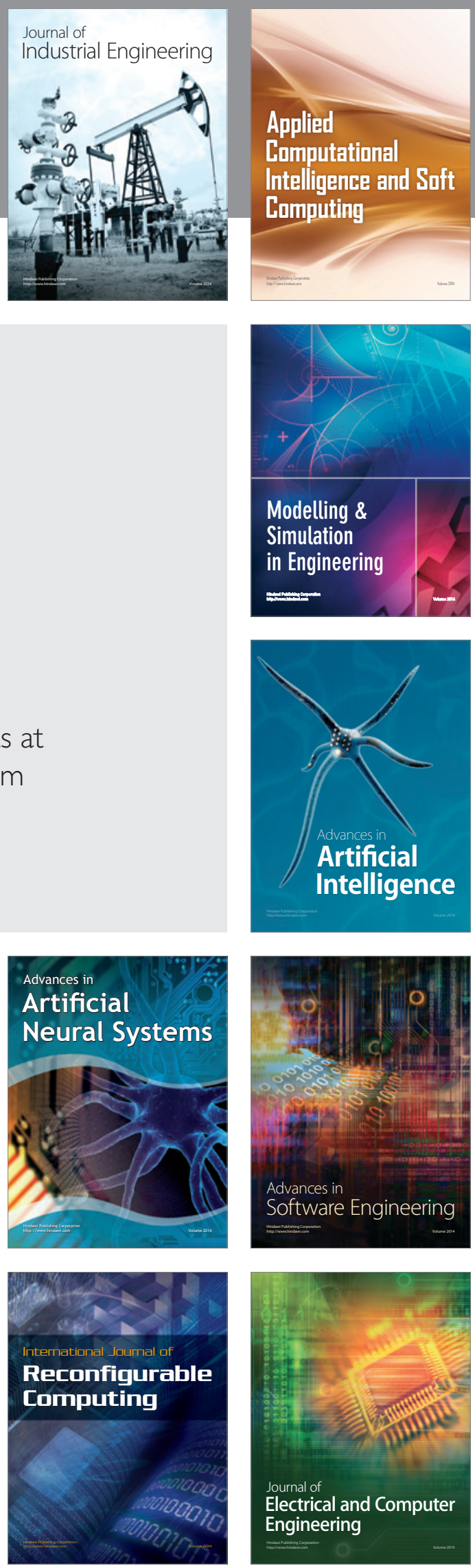